\title{
8 \\ The Nature of Jupiter's Magnetodisk Current System
}

\author{
N. Achilleos
}

\begin{abstract}
This chapter gives an overview of the properties of the magnetodisk current in the Jovian system. We describe the global morphology of the current sheet embedded in the plasmadisk / magnetodisk and the observational signatures of currents in this structure. We then consider the role of disk currents in force balance and plasmasheet structure in an axisymmetric, rotating system. We also describe the dependence of current density on spatial location, global size of the magnetosphere, and asymmetries plausibly associated with the influence of solar wind. We conclude with a simplified description of the microscopic nature of the particle motions in the magnetospheric plasma, whose collective action produces the currents themselves.
\end{abstract}

\subsection{INTRODUCTION}

A prominent feature of the giant, rapidly rotating magnetospheres of planets such as Jupiter and Saturn is the presence of a disklike body of plasma lying close to the magnetic equator of the planet itself. In the middle magnetosphere region (radial distances $\sim 10-40 \mathrm{R}_{\mathrm{J}}, 1$ $\mathrm{R}_{\mathrm{J}} \approx 71500 \mathrm{~km}=$ Jupiter radius), we may appeal to the approximation of the disk as a structure that has symmetry about the magnetic (dipole) equator of the planet. Since Jupiter's dipole moment is tilted at an angle of $\sim 10^{\circ}$ with respect to its rotation axis, it follows that the magnetic field in this region takes the form of a tilted, rotating structure. The plasma situated at different radial distances within this disk rotates at different angular velocities, the general trend being a decline in angular velocity from the initial, planetary value as one moves outward in the radial direction. The forces on this plasma

Department of Physics and Astronomy, Centre for Planetary Sciences, University College London, London, UK disk are related to the currents that flow both radially and azimuthally throughout it.

This chapter gives an overview of the properties of the magnetodisk current in the Jovian system. We begin with a description of the global morphology of the current sheet embedded in the plasmadisk/magnetodisk and the observational signatures of both azimuthal and radial flowing currents in this structure. We then consider the conceptual link between disk current, force balance, and plasmasheet structure in an axisymmetric, rotating plasmadisk. As part of these descriptions, we will also build a picture of the general behavior of the current density and its dependence on factors such as spatial location with respect to the planet, global size of the magnetospheric system, and asymmetries plausibly associated with the influence of the solar wind. We conclude with a description of the microscopic nature of the particle motions in the magnetospheric plasma, whose collective action produces the currents themselves. The specific descriptions in this chapter are also relevant to the comparison of planetary current systems by Khurana (Chapter 2, this

Electric Currents in Geospace and Beyond, Geophysical Monograph 235, First Edition.

Edited by Andreas Keiling, Octav Marghitu, and Michael Wheatland.

(C) 2018 American Geophysical Union. Published 2018 by John Wiley \& Sons, Inc. 


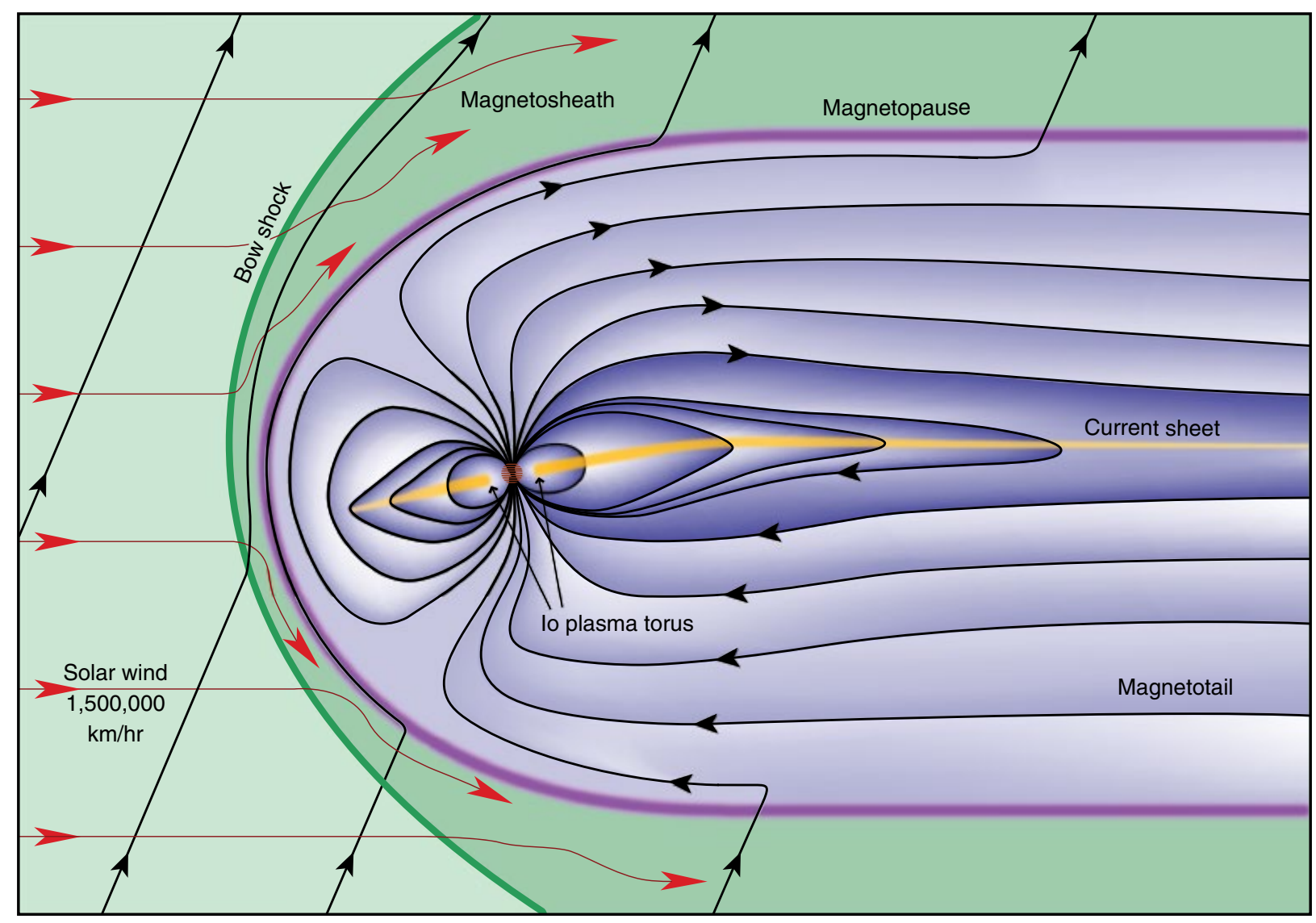

Figure 8.1 Noon-midnight cross section of Jupiter's magnetosphere. Image credit: Steve Bartlett and Fran Bagenal.

volume) and the discussion of giant planet current sheets by Arridge and Martin (Chapter 12, this volume).

\subsection{GLOBAL MORPHOLOGY OF THE JOVIAN CURRENT DISK}

The global structure of the main current system with which we are concerned is illustrated by the diagram of the Jovian magnetosphere in Figure 8.1. The highlighted current sheet region emphasizes some important aspects of the structure and orientation of the plasmasheet/magnetodisk, which carries this current. Figure 8.1 also shows the enormity of Jupiter's magnetosphere, which extends on the dayside to common distances near $\sim 62$ and $\sim 93 \mathrm{R}_{\mathrm{J}}$ [Joy et al., 2002]. We may investigate the equatorial magnetosphere, which is dominated over much of its radial extent by the magnetodisk current sheet, in terms of three broad regions, as follows [e.g., Khurana et al., 2004].

\subsubsection{Io Torus}

The Jovian magnetosphere's principal source of plasma is the volcanic satellite Io, which orbits the planet at a distance of $\sim 6 \mathrm{R}_{\mathrm{J}}$, and adds on the order of $\sim 1000 \mathrm{~kg} \mathrm{~s}^{-1}$ of sulphur and oxygen plasma to the system [Bagenal and Delamere, 2011; Hill et al., 1983]. The ensuing cold/warm plasma torus near Io's orbit, containing ions of temperatures 2-60 eV [Bagenal and Sullivan, 1981; Bagenal et al., 1985; Scudder et al., 1981], is aligned along the centrifugal equator (those points along the local dipolar field lines farthest from the planet's rotation axis). The torus contains on the order of $\sim 10^{6}$ tonnes of plasma and extends between $\sim 5-10 R_{J}$ with scale height on the order of $\sim 1 R_{J}$. Plasma is radially transported outward from the torus through processes such as flux tube interchange and magnetic field ballooning/reconfiguration (e.g., Thomas et al. [2004]; Kivelson and Southwood [2005]; review by Achilleos et al. [2015]; and references therein).

\subsubsection{Middle Magnetosphere}

The middle magnetosphere is the main focus for our description, and extends between $\sim 10-40 \mathrm{R}_{\mathrm{J}}$ in the equatorial plane. It is the region within which the magnetodisk plasma generally lies close to the dipole equator and also shows significant departure from corotation with the planet (i.e., the internal field). The latter behavior arises because of the finite conductance 
of 'that part of' the ionosphere which is magnetically conjugate to this region, and the decline of equatorial magnetospheric field strength with radial distance. Finite conductance is a principal factor that limits the radial disk current [e.g., Cowley and Bunce, 2001; Hill, 1979]. Since the equatorial field strength decreases with increasing radial distance, these combined factors limit the azimuthal Lorentz $(\mathbf{J} \times \mathbf{B})$ force, which acts to accelerate outflowing plasma toward planetary corotation. Beyond the distances mentioned, the plasma thus lags the planet with respect to angular velocity. This subcorotation is a key concept in the theory of magnetosphere-ionosphere coupling of Hill [1979]. The radial current system is closed by ionospheric currents as well as field-aligned currents, some of which correspond to auroral particle precipitation (e.g., Cowley and Bunce [2001]; Nichols and Cowley [2004]; Ray et al. [2012]; Ray and Ergun [2012]; Chapter 21 in this volume by Ray and references therein).

The plasma in the middle magnetosphere generally consists of a low-energy "warm" component $(\lesssim 100 \mathrm{eV})$ originating from outward diffusion of Io torus plasma, and an energetic "hot" component $(\gtrsim 20 \mathrm{keV})$, which is thought to be accelerated/heated torus plasma interspersed with plasma from the outer magnetosphere moving inward (e.g., Siscoe et al. [1981]; Kivelson et al. [1997]; Thorne et al. [1997]; Bagenal and Delamere [2011]).

Let us now consider the azimuthal currents in the plasmadisk. In the middle magnetosphere, these currents generate perturbation magnetic fields, which become comparable to the internal field of the planet beyond $\sim 20 \mathrm{R}_{\mathrm{r}}$. In Connerney et al. [1981]'s magnetic field model for an axisymmetric current disk, the current flows azimuthally within an equatorial disk of uniform thickness $2 \mathrm{D}$, extending between radii $\rho_{1}$ and $\rho_{2}$, and in which the current density $J_{\varphi}$ is assumed positive everywhere (i.e., in the direction of planetary rotation) with radial dependence $J_{\varphi} \propto \frac{1}{\rho}$. By tilting the equatorial plane of the model to match the position of the Jovian dipole equator, Connerney et al. [1981] were able to reasonably fit some of the Voyager and Pioneer magnetic observations of the perturbation field (measured field minus a model internal field), for radial distances $\lesssim 30 \mathrm{R}_{\mathrm{J}}$ (Fig. 8.2 shows an example).

(a)

Voyager I Jupiter perturbation field 48-Sec avg

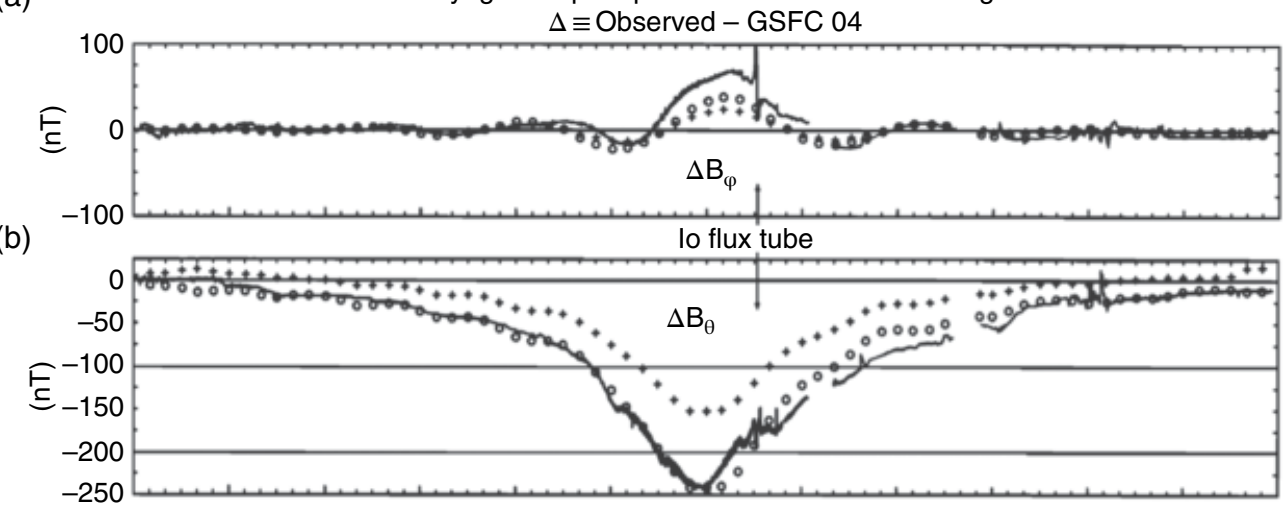

(c)

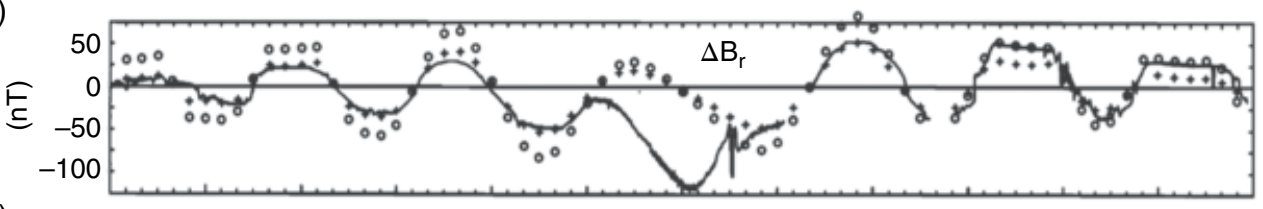

(d)

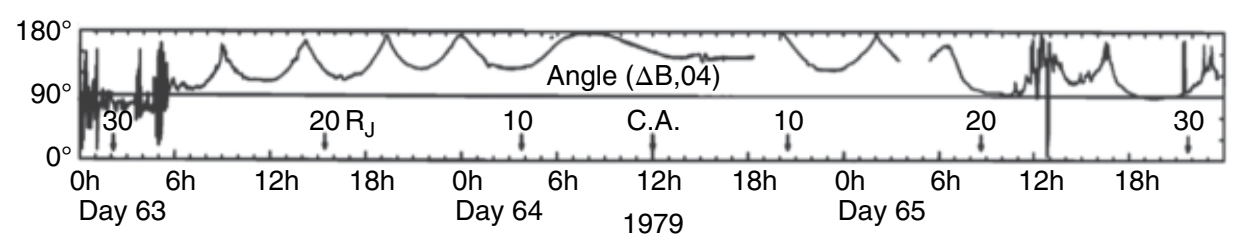

Figure 8.2 Voyager 1 observations of the Jovian perturbation field (estimated using the $\mathrm{O} 4$ internal field model), with corresponding predictions from two disk current models of the same vertical thickness $\left(2 D=5 R_{\mathrm{J}}\right)$ and inner radius $\left(\rho_{1}=5 \mathrm{R}_{\jmath}\right)$, but differing outer radius and current density (circles: $\rho_{2}=50 R_{J}, J_{1}=5.1 M A R_{J}^{-2}$ at $\rho_{1}$; crosses: $\rho_{2}=30 R_{J}, J_{1}=3.42 M A R_{J}^{-2}$ ). The field components (top three panels: (a) azimuthal, (b) meridional, (c) radial) are in a spherical polar system. The bottom panel $(\mathrm{d})$ shows time-dependent spacecraft radial distance and angle between the perturbation field vector $\Delta B$ and O4 model. From Connerney et al. [1981]. 
The observed oscillating radial $\left(\Delta B_{r}\right)$ and azimuthal $\left(\Delta B_{\varphi}\right)$ field components in Figure 8.2 are signatures of the rotating, tilted current sheet moving over the spacecraft twice per planetary rotation. Both of these components pass through zero as the spacecraft moves relative to the sheet, from the northern $\left(\Delta B_{r}>0, \Delta B_{\varphi}<0\right)$ to the southern $\left(\Delta B_{r}<0, \Delta B_{\varphi}>0\right)$ magnetic hemisphere. The $\Delta B_{r}$ reflects the radially stretched field (Fig. 8.1), in which the inward magnetic curvature force balances the outward forces in this region from the radial plasma pressure gradient and the centrifugal force (see section 8.3). The $\Delta B_{\varphi}$ observations generally display the azimuthal bendback of field lines from the poloidal geometry of the Connerney et al. [1981] model; this is the effect of the radial corotation enforcement currents that flow in the disk. Appropriate detailed analysis of $\Delta B_{\varphi}$ observations may be used to estimate the Io mass-loading rate [Khurana and Kivelson, 1993].

\subsubsection{Physical Origin of Asymmetry in the Current Disk}

The planar, axisymmetric approximation is less suitable for describing the morphology of the current disk at distances beyond $\sim 20-30 \mathrm{R}_{\mathrm{r}}$. At these larger distances, centrifugal and pressure-related forces become important influences on the stress balance in the system (see section 8.3). As a result, the equilibrium current sheet is located between the dipole and centrifugal equators. Khurana [1992] also demonstrated that the sheet in the distant magnetotail $\left(\gtrsim 60 \mathrm{R}_{\mathrm{J}}\right)$ develops a hinging and becomes parallel to the direction of solar wind flow outside the magnetosphere. Stress associated with the solar wind dynamic pressure is communicated to the plasma inside the magnetosphere via the fringing field produced by forces acting at the magnetopause boundary.

A very simple example of this type of communication is illustrated by the equatorial magnetic field $\Delta B$ generated by magnetopause currents in a simplified, axisymmetric model of the magnetodisk region (see section 8.3). The model considered later with radius $60 \mathrm{R}_{\mathrm{J}}$ has an equatorial ratio $\Delta B / B_{e q}$ of magnetopause field to total field, which varies with radial distance. The $\Delta B / B_{e q}$ increases with distance from the planet, exceeds $\sim 20 \%$ beyond $\sim 20 \mathrm{R}_{\mathrm{J}}$, and attains a value $\sim 40 \%$ just inside the magnetopause. In this example, the magnetopause fringing field thus contributes significantly to the total field strength even in the middle magnetosphere, and becomes stronger for a more compressed system. In the more general case, the fringing field may also support strong rotation or hinging of the magnetospheric field in the neighborhood of the magnetopause itself.

In addition to this hinging effect, systematic delays in the expected arrival times of a planar current sheet are observed in this region. This phenomenon arises partly because of the finite propagation time of the Alfvén waves launched from the ionosphere, which communicate information about the rocking of the rotating, tilted planetary dipole to the distant magnetosphere. Current sheet delays are also controlled by the strong bending of the field lines out of meridian planes, against the direction of planetary rotation, which arises from the azimuthal stresses on the plasma. Field lines at Jovian dawn, for example, can be bent out of their near-planet meridian planes by angles $\lesssim 40^{\circ}$ for distances $\lesssim 100 \mathrm{R}_{\mathrm{J}}$ [Khurana et al., 2004]. It is important to note that the frequency of current sheet encounters at any given distance is controlled by the period of the planet. The rotating dipole ultimately imposes a modulation pattern that influences the vertical location of the magnetospheric plasma with respect to the rotational equator. Thus, while outer magnetospheric disk plasma subcorotates with respect to the planet, the vertical location of maximum density along a subcorotating flux tube is variable, and partly determined by the rotational phase of the planetary dipole.

To represent these types of hinging and delay effects, Khurana [1992] developed a "hinged-magnetodisk" model. In an earlier study, Behannon et al. [1981] introduced hinging and delay effects into their axisymmetric model of the current sheet surface. Khurana [1992] presented a model in which the hinging was linked to solar wind forcing, thus breaking axial symmetry, and was able to improve the quality of the model fit to Pioneer and Voyager observations. His formula for the altitude $Z_{C S}$ of the current sheet with respect to the Jovigraphic equator was:

$$
Z_{C S}=\rho \tan \left(\theta_{d i p}\right)\left(\frac{x_{o}}{x} \tanh \left(\frac{x}{x_{o}}\right) \cos (\lambda-\delta)\right)
$$

In this equation, $\rho$ is cylindrical radial distance (with respect to the planet's rotational axis) and $\theta_{d i p}$ is the dipole tilt. The hinging is implemented through the coordinate $x$ along the Jupiter-Sun axis, and the hinge distance $x_{o}$ indicates where hinging produces significant departure from a planar structure. The $\lambda$ represents longitude, and the harmonic term introduces a delay in the azimuthal current sheet position by making use of $\delta=\delta_{o}-\left(\Omega_{J} \rho_{o} / v_{o}\right) \ln \cosh \left(\rho / \rho_{o}\right)$, where $\delta_{o}$ is the longitude of the plane containing the dipole, $\Omega_{J}$ is the planet's angular velocity of rotation, $\rho_{o}$ is the distance at which delay effects cause significant departure with respect to a planar current sheet, and $v_{o}$ can be interpreted as a wave velocity that carries information about dipole orientation into the outer magnetosphere. Figure 8.3 illustrates some of these effects by presenting maps of $Z_{C S}$ from the point of view looking from the north onto the Jovigraphic equatorial plane. We show two examples of the model 
(a)

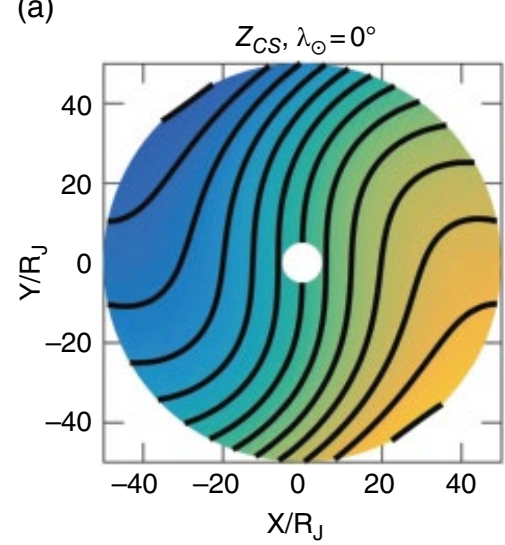

(b)

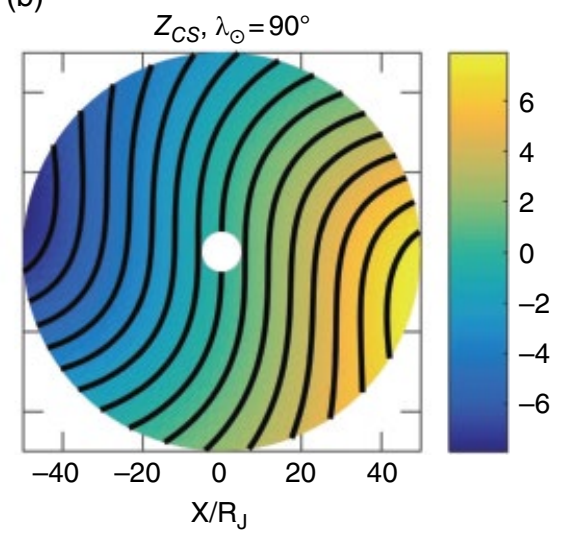

Figure 8.3 Color-coded plots of current sheet altitude $Z_{C S^{\prime}}$ with respect to the Jovigraphic equator (XY plane), calculated using the hinged-magnetodisk formulae of Khurana [1992] for projected radial distances $\rho=5-50 \mathrm{R}_{\mathrm{J}}$. The projection of the northern planetary dipole lies along the $-\mathrm{X}$ axis in both panels, while the planet-Sun direction is at a longitude of $\lambda_{\odot}=0^{\circ}$ (positive $X$ direction) and $90^{\circ}$ (positive $Y$ direction) in the respective left (a) and right (b) panels. The planet's rotational axis is assumed, for simplicity, to be orthogonal to the XY plane (the actual obliquity of this axis is small, $\sim 3^{\circ}$ ). The black curves are contours of constant $Z_{C S}$ spaced by $1 R_{\mathrm{J}}$ (with $Z_{C S}=0$ contours passing through plot centers), and illustrate the strong hinging associated with the $\lambda_{\odot}=0^{\circ}$ configuration. The azimuthal bending of these contours in the outer region of the sheet produces the observed current sheet delay effects described in the text. Values for the model parameters (see text) used for these plots were taken from Khurana [1992].

current sheet, for configurations where the Jupiter-Sun direction is parallel and orthogonal to the dipole longitude meridian. The former case shows the most pronounced hinging. We used the following geometrical parameters for the plots in Figure 8.3, identical to those from the fit of Khurana [1992]: $x_{o}=33.5 \mathrm{R}_{\mathrm{y}}, \rho_{o}=33.2 \mathrm{R}_{\mathrm{s}}, v_{o}=37.4 \mathrm{R}_{\mathrm{J}} / \mathrm{hr}$.

Magnetic field models that incorporate azimuthal bendback into the field lines can be constructed using the approach where the field is expressed in terms of two Euler potential functions, $f$ and $g$ :

$$
\mathbf{B}=\nabla f \times \nabla g .
$$

Without loss of generality, if the Euler potential $g$ is, for example, a linear function of azimuthal angle $\varphi$ (magnetic longitude), we obtain a poloidal field structure, where all field lines lie in a single plane. This poloidal class of Euler-potential field models has been combined with the force-balance condition to obtain equilibrium models for both field and plasma distribution (see section 8.3 for further details).

Goertz et al. [1976] used an Euler potential with dependence on both $\rho$ and $\varphi$ to create nonpoloidal field lines with bendback. Khurana [1997] further developed this type of field model for the current sheet by using Euler potentials suitable for coupling the current sheet field to a model of Jupiter's internal magnetic field. He included current sheet delay and hinging by making the Euler potentials dependent on $Z_{C S}$, the altitude of the current sheet (see equation [8.1]).
We conclude this section on Jovian current sheet asymmetry by summarizing the local-time asymmetry in the intensity of the magnetodisk currents, which was found by Khurana [2001], from an analysis of magnetometer data from six spacecraft to have visited the Jovian system, including Galileo. The height-integrated radial and azimuthal current densities flowing in the current sheet $\left(J_{\rho}^{\prime}\right.$ and $\left.J_{\varphi}^{\prime}\right)$ were calculated in this study according to the following formulae, which essentially arise from Ampère's law applied across the vertical thickness of the sheet:

$$
\begin{gathered}
\mu_{o} J_{\rho}^{\prime}=-2 \Delta B_{\varphi, l} / \mu_{o} \\
\mu_{o} J_{\varphi}^{\prime}=\left(2 \Delta B_{\rho, l}-2 W \frac{\partial}{\partial \rho}\left(\Delta B_{z}\right)\right)
\end{gathered}
$$

where $\Delta B_{\varphi, l}$ and $\Delta B_{\rho, l}$ are the respective perturbation fields in the cylindrical radial and azimuthal directions, measured just outside the current sheet (lobe region); $\Delta B$ is the vertical perturbation field; and $W$ is the half-thickness of the sheet. $W$ is not constant; it may vary according to solar wind dynamic pressure (see section 8.3) and local time. For example, Khurana et al. [2004] present Galileo magnetic data in the outer magnetosphere $\left(35-55 \mathrm{R}_{\mathrm{J}}\right)$ that indicate that the dawn-side half-thickness is $\sim 1.5 \mathrm{R}_{\mathrm{J}}$ - thin compared to the inferred duskside value of $\sim 7.6 \mathrm{R}_{\mathrm{f}}$.

The resulting profiles of $J_{\rho}^{\prime}$ and $J_{\varphi}^{\prime}$ as a function of local time are shown in Figure 8.4, from Khurana et al. 

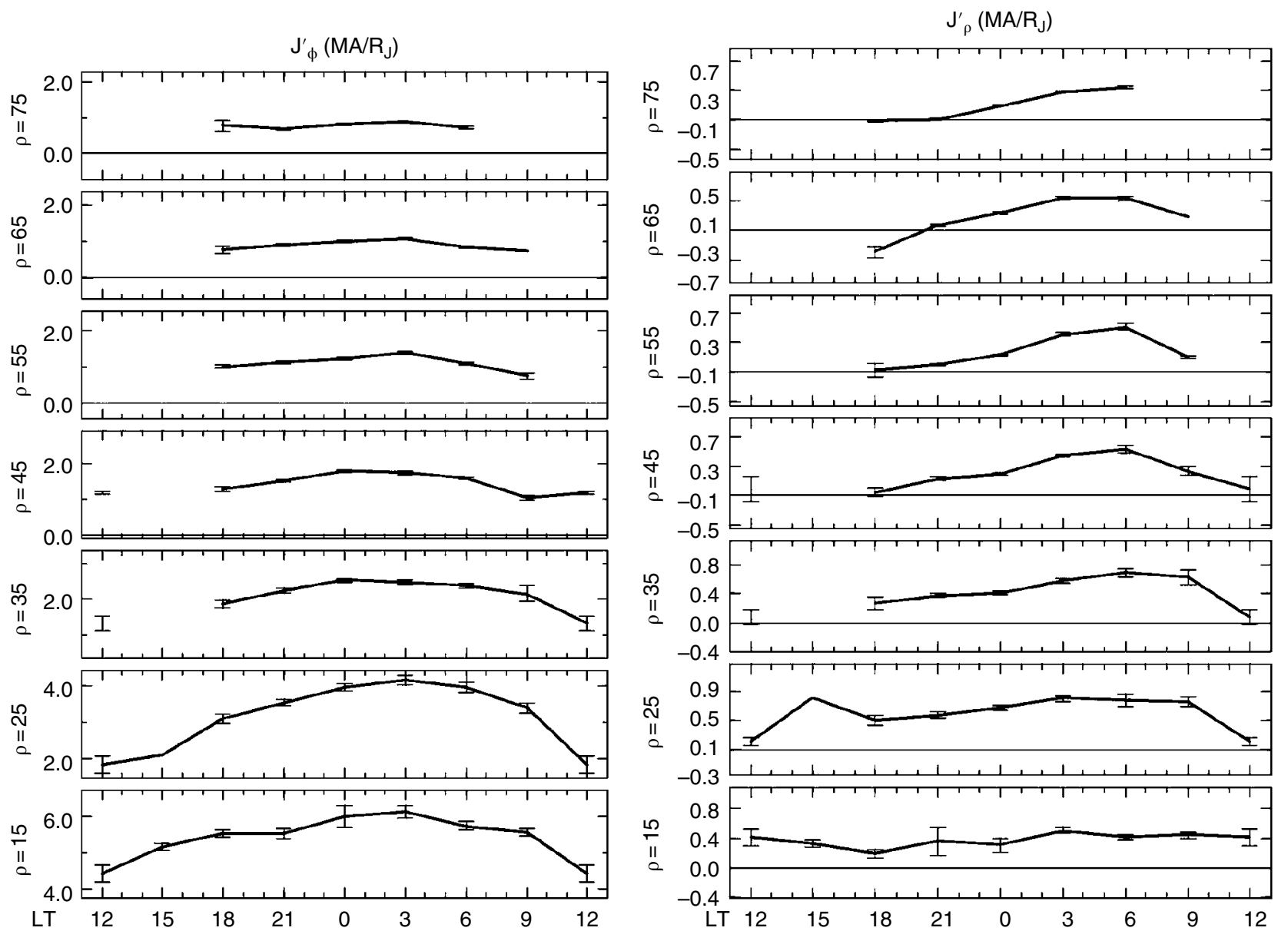

Figure 8.4 Binned profiles, derived from magnetic data, of the height-integrated radial and azimuthal current density in the current sheet, as a function of local time. Profiles for different radial distance intervals are shown, according to the vertical axis labels. From Khurana et al. [2004], adapted from Khurana [2001].

[2004] (adapted from Khurana [2001]). There is a daynight asymmetry in the azimuthal current density: the total azimuthal current flowing on the nightside between distances $10-50 \mathrm{R}_{\mathrm{J}}$ is $\sim 144 \mathrm{MA}$, compared with the corresponding dayside current, integrated over the same distances, of $\sim 88 \mathrm{MA}$. Current continuity thus requires the existence of field-aligned current systems at dusk and dawn that respectively feed and drain the nightside partial ring current. This type of current system is analogous to the terrestrial Region 2 currents, associated with solar wind-driven convection. It thus seems plausible that the solar wind may be modulating the local-time structure of the middle magnetosphere at Jupiter.

Another important property of the current profiles in Figure 8.4 is the occurrence of negative (planetward) values of $J_{\rho}^{\prime}$, especially at distances near $\sim 65 \mathrm{R}_{\mathrm{J}}$ in the dusk sector. This finding indicates that, beyond $\sim 65 \mathrm{R}_{\mathrm{f}}$, the relatively simple picture of a subcorotating magnetodisk breaks down. Excellent reviews of global plasma distribution and flow may be found in, for example,
Vasyliūnas [1983], Kivelson and Southwood [2005], and Bagenal and Delamere [2011].

\subsubsection{Outer Dayside Magnetosphere}

Jupiter's dayside current sheet must, of course, be finite in radial extent, but to what distances does it reach? The magnetopause certainly represents a generous outer boundary for the magnetodisk. In fact, spacecraft observations have revealed that the magnetodisk current sheet does not extend all the way to the magnetopause. In the morningto-postnoon sector, there is a region known as the "cushion," with radial extent of order $\sim 20 \mathrm{R}_{\mathrm{J}}$ (near noon), lying between the outer edge of the plasmadisk and the magnetopause, where the field shows strong fluctuations and is mainly southward directed [Kivelson and Southwood, 2005; Smith et al., 1974]. Magnetic nulls are sometimes present in the cushion: these are a likely signature of bubbles of plasma detaching from the edge of the plasmadisk [Kivelson and Southwood, 2005; Southwood et al., 1993]. 


\subsection{FORCE-BALANCE MODELS OF THE MAGNETODISK}

Caudal [1986] developed a model of the Jovian magnetodisk, based on the assumption of global force balance between the Lorentz force, plasma (isotropic) pressure gradient, and centrifugal force in an axisymmetric system with parallel rotational and magnetic (planetary dipole direction) axes. This condition may be written as:

$$
\mathbf{J} \times \mathbf{B}-\nabla P+n_{i} m_{i} \rho \omega^{2} \mathbf{e}_{\rho} \approx 0
$$

where the symbols have the following meanings: current density $\mathbf{J}$, magnetic field $\mathbf{B}$, plasma pressure $P$, ion number density $n_{i}$, ion mass $m_{i}$, cylindrical radial distance $\rho$, plasma angular velocity $\omega$, and cylindrical radial unit vector $\mathbf{e}_{\rho}$. Caudal [1986] showed that this physical condition was equivalent to a differential equation relating the spatial gradients of the magnetic field (radial and meridional) to a "source function" $g(r, \theta)$, which represents the influence of the plasma properties on the field structure. The $g(r, \theta)$ can then be determined throughout the magnetospheric volume from its equatorial values (constructed from observations) by using the forcebalance formalism and model assumptions (e.g., uniform temperature and effective ion mass along a field line). Recent adaptations of Caudal [1986]'s model have been presented for the Kronian magnetosphere [Achilleos et al., 2010a; Achilleos et al., 2010b] and for the Jovian magnetosphere [Nichols, 2011; Nichols et al., 2015].

This type of model is useful for illustrating how the plasmadisk structure responds to changes in magnetospheric size. In Figure 8.5, we show the computed pressure of the cold plasma population from an implementation of Caudal [1986]'s Jovian magnetodisk model, computed for two different equatorial radii 60 and $90 \mathrm{R}_{\mathrm{J}}$. Magnetic field lines anchored to the same planetary surface footpoints are also shown in both panels, as well as the $\beta_{c}=0.2$ contour of cold plasma beta, which represents a plasmasheet boundary. The plasmasheet in the middle magnetosphere is thinner for the expanded model. This behavior is linked to the corresponding change in the shape of the field lines, which become significantly more disklike (radially distended) in the expanded case. In these models, cold plasma pressure along a given field line decreases, relative to the equatorial value $P_{o}$, according to:

$$
P=P_{o} \exp \left(\frac{\rho^{2}-\rho_{o}^{2}}{2 l^{2}}\right)
$$

where $\rho_{o}$ is the cylindrical radial distance of the equatorial point of the field line and $l$ is a pressure scale length given by $l=2 k_{B} T /\left(\mathrm{m}_{\mathrm{i}} \omega^{2}\right)$, where $k_{B}$ is Boltzmann's constant and $T$ is temperature. The points on the field line with cylindrical distance $\rho_{\mathrm{o}}-l$ are thus located at a smaller vertical distance from the equator for a more disklike field, leading to a thinner plasmasheet. A centrifugally confined plasma, situated on flux tubes with equatorial radii $\rho_{o}$, would satisfy the condition $l^{2} \ll<\rho_{o}^{2}$, which, using the definition of $l^{2}$, is equivalent to $k_{B} T<<\frac{1}{2} m_{i} \omega^{2} \rho_{o}^{2}$, that is, the typical thermal energy of the cold ions in the disk is small compared to their kinetic energy of bulk rotation. Some representative, equatorial values of $l$ according to the $60 \mathrm{R}_{\mathrm{J}}$ magnetodisk model (which incorporates observed values of relevant plasma parameters) are $l=\sim 3, \sim 8$, and $\sim 40 \mathrm{R}_{\mathrm{J}}$ at respective radial distances of 10,30 , and $60 \mathrm{R}_{\mathrm{J}}$.

Figure 8.6 shows the azimuthal current density for the same magnetodisk models used for Figure 8.5. We show the total current density from both hot and cold plasmas. The total azimuthal current that flows across the entire meridian is $\sim 270$ MA and $\sim 310$ MA for the respective compressed and expanded models. The corresponding magnetic moments of these extensive current systems are $\sim 1.7 \mu_{J}$ and $\sim 3.3 \mu_{J}$, which exceed the magnetic moment $\mu_{J}$ of the planet's dipole field (defined $\mu_{J}=4 \pi B_{J} R_{J}^{3} / \mu_{o}$, where $B_{J} \approx 428000 \mathrm{nT}$ is the equatorial magnetic field strength at the planet's surface). These total currents are similar in magnitude to those flowing through the

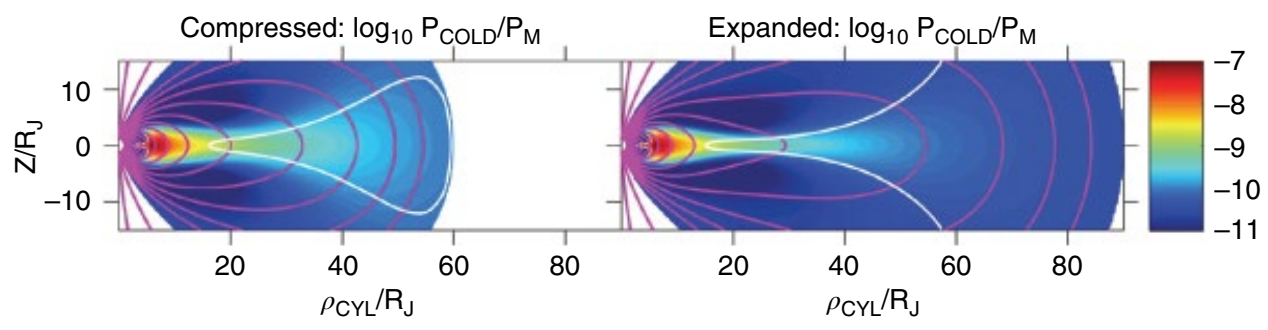

Figure 8.5 Magnetic meridians from compressed and expanded force-balance models of the Jovian magnetodisk (see text). Logarithm of normalized cold plasma pressure is indicated by the color scale (the pressure normalization factor is $P_{M}=B_{J}^{2} / \mu_{0}$, where $B_{J}=428000 \mathrm{nT}$ is the approximate equatorial surface field strength at Jupiter). The solid magenta curves are magnetic field lines and the white curves are the loci defined by cold plasma beta $\beta_{\mathrm{c}}=0.2$. 


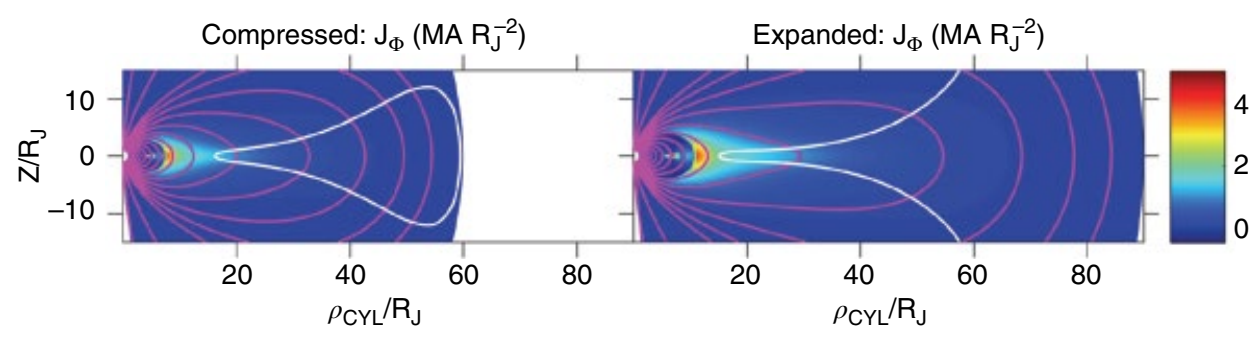

Figure 8.6 As for Figure 8.5, but with the color scale now showing total azimuthal current density (from both cold and hot plasmas), in units of $M A R_{\jmath}^{-2}$.

Connerney et al. [1981] current disk models described in Figure 8.2 ( 150-290 MA). Not visible at the scale of these plots is the region of weak, negative (i.e., flowing against the direction of planetary rotation) current found in the inner magnetosphere (at distances $\lesssim 7 R_{\mathrm{J}}$ ). Negative current arises in regions where the component of force acting on the plasma in the direction orthogonal to the magnetic field, due to pressure gradients plus centrifugal force, is inward directed.

Nichols et al. [2015] has recently developed the first Caudalian model of the Jovian magnetodisk to include the effects of anisotropic hot plasma pressure. Observations by Paranicas et al. [1991] of the anisotropic particle pressure (for particle energies $\gtrsim 28 \mathrm{keV}$ ) were used to constrain the ratio $P_{\|} / P_{\perp}$ of pressures parallel and perpendicular to the magnetic field. This ratio reaches values up to $\sim 1.2$ at the model equator. Force balance equations were then numerically integrated to produce models of the magnetodisk as a function of a hot plasma parameter $K_{h}$, which is the product of hot plasma pressure and flux tube volume in the outer magnetosphere, and constrained using spacecraft observations. Figure 8.7 shows some of the results of this study: specifically, maps of the contributions to current density, which balance the different forces on the plasma, and the geometry of the magnetic field lines. The hot plasma pressure gradient is balanced by the strongest contribution to the current overall but note also that the hot plasma anisotropy current also rapidly increases with $K_{h}$. The increased radial stretching of the field with $K_{h}$ generally confines the currents closer to the equatorial plane, and also reduces the magnitude of the local hot plasma pressure gradient.

The calculations by Nichols et al. [2015] also have important implications for auroral morphology and magnetodisk stability. With the inclusion of hot plasma pressure anisotropy, the observed few-degree latitudinal shifts of the modeled main oval and Ganymede footprint, arising from realistic changes in $K_{h}$, are comparable to the analogous shifts observed in auroral images (e.g., Grodent et al. [2008]; Bonfond et al. [2012]). In addition, the model's middle magnetosphere attains equatorial values of $\beta_{h, \|}-\beta_{h, \perp}$ (difference between parallel and perpendicular hot plasma beta) of $\sim 1-2$, for $K_{h}=2.0-2.5 \times 10^{7} \mathrm{~Pa} \mathrm{~m} \mathrm{~T}^{-1}$. These values suggest that this region may be susceptible to the firehose instability (see also Kivelson and Southwood [2005], who discuss the "centrifugal parallel heating" mechanism by which this instability may arise at the equatorial portions of rotating flux tubes, which simultaneously expand as they rotate into the dusk sector). Indeed, for $K_{h}$ values larger than this range, $\beta_{h, \|}-\beta_{h, \perp}$ can exceed 2 near $\sim 25 \mathrm{R}_{\mathrm{J}}$ and the model does not converge. Nichols et al. [2015] point out that this result suggests that small-scale release, or drizzle, of Iogenic plasma may commonly arise from the Jovian middle magnetosphere under conditions of adequately high hot plasma pressure (see also Bagenal [2007]).

In summary, the inclusion of pressure anisotropy in a Jovian magnetodisk model, as described by Nichols et al. [2015], results in the following main modifications when compared with the predictions of isotropic-pressure models: (1) Radial force associated with anisotropy plausibly promotes firehose instability in the middle magnetosphere, possibly associated with an additional channel of mass loss. (2) Anisotropy enhances the distortion of the magnetic field lines, and greatly facilitates few-degree shifts in the main auroral latitude, compatible with those observed.

\subsection{MICROSCOPIC NATURE OF THE DISK CURRENT}

In general terms, the relation between the magnetic field flux density B (commonly referred to in space physics as the "magnetic field"), the free current density $\mathbf{J}_{\mathbf{f}}$, and the plasma magnetization $\mathbf{M}$ is:

$$
\nabla \times \mathbf{B}=\mu_{o}\left(\mathbf{J}_{\mathbf{f}}+\nabla \times \mathbf{M}\right)
$$

where we recognize that the total source of the vector field $\mathbf{B}$ comprises both free and magnetization current density, the latter being equal to $\nabla \times \mathbf{M}$. Free current is associated with drift motions, which displace the guiding centers of particles, while magnetization current can arise from the collective action of many particle 


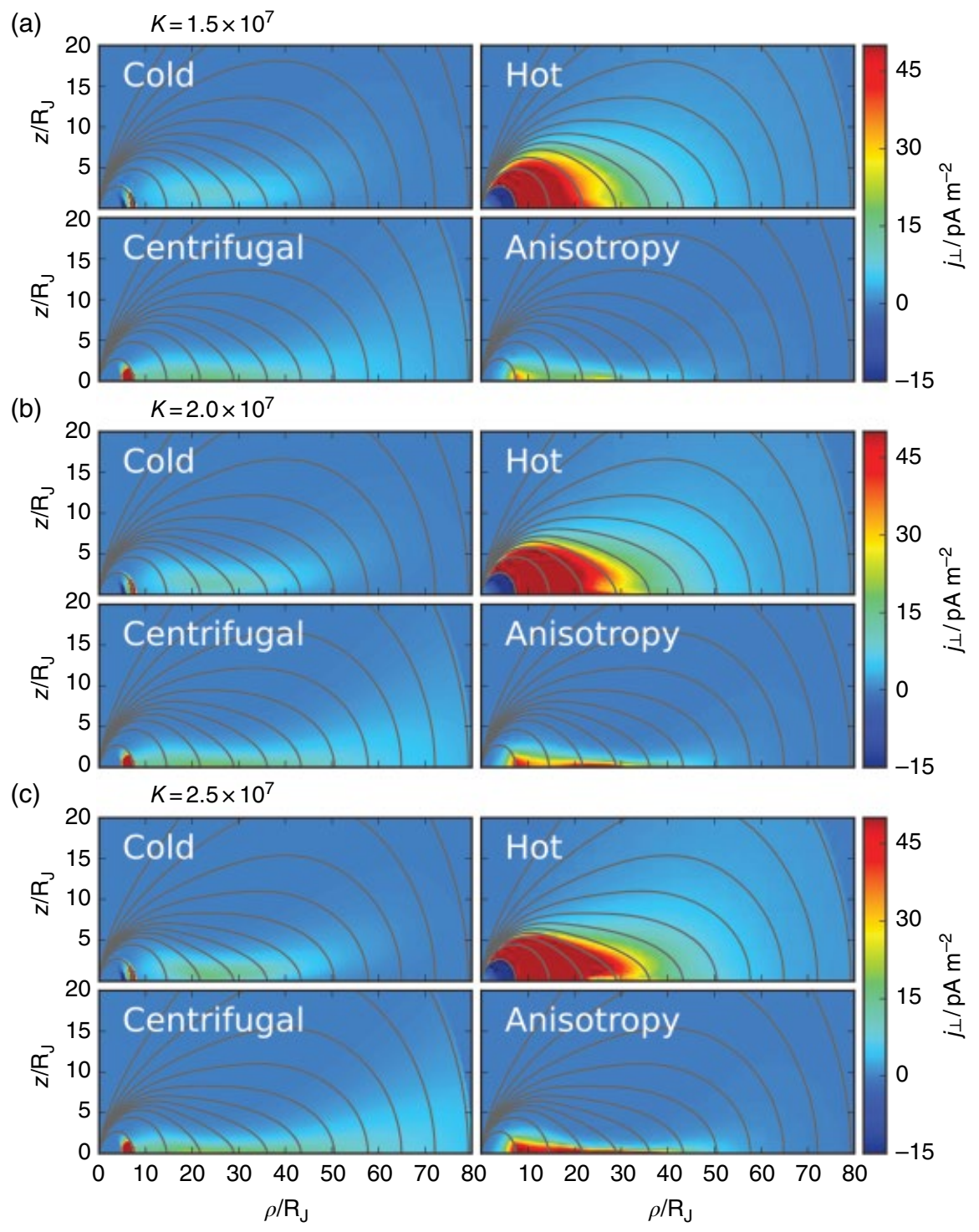

Figure 8.7 Magnetodisk models showing current density on a color scale and magnetic field lines as solid curves. Model outputs associated with the different forces on the plasma (pressure gradients for cold and hot populations, centrifugal force, anisotropy force) are shown. Three sets of models are shown, labeled with the corresponding hot plasma parameter $K_{h}$ (see text) used for the calculations. From Nichols et al. [2015].

gyrations, even in a plasma where the guiding centers are stationary (provided the curl of the magnetization is nonzero).

We may further investigate the relative intensities of the two types of current in a steady-state system by adapting the example described by Gurnett and Bhattacharjee [2005]. For simplicity, we consider a cylindrically symmetric system with magnetic field everywhere directed parallel to the axis of symmetry. The magnetic moment of the plasma per unit volume is:

$$
\mathbf{M}=\left(-N W_{g} / B\right) \hat{\mathbf{B}}
$$

where $N$ is the total number density of particles in the isotropic plasma with mean kinetic energy of gyration $W_{g}$ per particle, and $\hat{\mathbf{B}}$ denotes a constant unit vector along the direction of the magnetic field. The magnetization current density, $\mathbf{J}_{\mathbf{m}}=\nabla \times \mathbf{M}$, can then be evaluated in this picture:

$$
\mathbf{J}_{\mathbf{m}}=\left(\frac{-1}{B^{2}}\right) \nabla P \times \mathbf{B}+\left(\frac{P}{B^{3}}\right) \nabla B \times \mathbf{B}
$$

where we have used the definition for isotropic plasma pressure $P=N W_{g}$. 
Consider a simple plasma with particles of equal gyroradius $r_{g}$ and threaded by a uniform magnetic field in the $z$ direction. Equation (8.9) reveals that, in this case, the magnetization current density reduces to the first term on the right-hand side, which is often referred to as "diamagnetic current density." Assume now that all the particles in our fictitious plasma have the same temperature, and all gyrate in the $x y$ plane, but that the guiding centers themselves are stationary. Along a reference line defined by $x=x_{o}$, the current depends on the motions of particles with guiding centers located within a distance $\sim r_{g}$ of the reference line. If the plasma has a density gradient in the $x$ direction, this implies that the number of gyrating particles crossing the line, which have guiding centers to its right, will not equal the corresponding number for particles with guiding centers to its left. This imbalance leads to a net current in the $y$ direction, despite the fact that the guiding centers are stationary.

The final term in equation (8.9) is equal to $-J_{g}$, where $J_{g}$ is the contribution to the current density due to magnetic gradient drift of the plasma particles. The other drift current in this example is the inertial current $J_{\Omega}$ associated with the bulk rotation of the plasma, which is given by:

$$
\mathbf{J}_{\Omega} \approx\left(\frac{N W_{\Omega}}{\rho B^{2}}\right) \mathbf{e}_{\rho} \times \mathbf{B}
$$

where $W_{\Omega}$ is the rotational kinetic energy per plasma ion, $\rho$ is cylindrical radial distance, and $\mathbf{e}_{\rho}$ is the cylindrical radial unit vector. This current may be pictured as arising from the drift associated with the centrifugal force in a corotating frame of reference. For a single ion of mass $m_{\mathrm{i}}$ and charge $q_{i}$, the centrifugal force per unit charge is simply $\left(2 W_{\Omega} /\left(q_{i} \rho\right)\right) \mathbf{e}_{\rho}$. The corresponding guiding center drift velocity will thus be $v_{\mathrm{d} \Omega}=\left(2 W_{\Omega} /\left(q_{i} \rho B^{2}\right)\right) \mathbf{e}_{\rho} \times \mathbf{B}$. Hence, the inertial current density carried by the rotating ions will be, for our quasi-neutral plasma, $J_{\Omega}=\left(N q_{i} / 2\right) v_{d \Omega}$, which is equal to the expression in equation (8.10). This will be a good approximation for the total inertial current density, since the mass of the electrons is negligible when compared with that of the ions.

Using the above expressions for current densities, we note that the total current density in this example is:

$$
\begin{aligned}
& \mathbf{J}_{\mathbf{t}}=\mathbf{J}_{\mathbf{m}}+\mathbf{J}_{\mathbf{g}}+\mathbf{J}_{\Omega}= \\
& \left(\left(\frac{-1}{B^{2}}\right) \nabla P+\frac{N W_{\Omega}}{\rho B^{2}} \mathbf{e}_{\rho}\right) \times \mathbf{B}
\end{aligned}
$$

The expression for $\mathbf{J}_{\mathbf{t}}$ indicates an important general principle: the drift and magnetization currents for a plasma in magnetostatic equilibrium combine to produce a total current density $\mathbf{J}_{t}$ for which the associated magnetic body force $\mathbf{J}_{\mathbf{t}} \times \mathbf{B}$ exactly balances the other forces on the plasma. In the present example, the other forces are the pressure gradient and the centrifugal volume force.

It also follows, in this case, that the magnitude of the magnetization current will be large compared to that of the drift current wherever both of the following local conditions are satisfied by the radial length scale $l_{P}$ of the plasma pressure:

$$
l_{P}<<l_{B}, \quad l_{P}<<\frac{W_{g}}{W_{\Omega}} \rho
$$

where $l_{B}$ is the length scale of the field strength. In a more general context, we would expect magnetization current to be dominant in a rotating plasma at natural boundaries where the pressure length scale is small in comparison to the minimum of: the length scale of the magnetic field strength, the radius of curvature of the field lines, and a length scale (see equation [8.12]) involving the ratio of the thermal to rotational energy of a plasma ion. This ratio also determines the length scale for pressure in a centrifugally confined plasma according to the theory of Caudal [1986] (see section 8.3).

A fully realistic quantitative comparison of magnetization and drift current for the Jovian system is beyond the scope of this review. Nevertheless, we may appeal to the predicted equatorial values of the ratio $l_{P} / l_{B}$ from our $60 \mathrm{R}_{\mathrm{J}}$ magnetodisk model in order to obtain a preliminary indication of where we would expect magnetization current to be dominant. When considering total plasma pressure, we find $l_{P} / l_{B}<0.1$, for example, at radial distances from $\sim 43 \mathrm{R}_{\mathrm{J}}$ to just inside the magnetopause. This behavior arises due to the very gradual (compared to pure dipole) decrease in equatorial field strength of the disklike field in the outer magnetosphere. When considering the cold plasma alone, we find that $0.5<l_{P} / l_{B}<1$ within the inner and outer edges of the Io plasma torus, themselves natural plasma boundaries with relatively strong spatial gradients in plasma pressure.

\subsection{SUMMARY}

A combination of data analysis and theoretical modeling related to Jupiter's magnetodisk has produced an ever more detailed picture of the system. Elements of this picture include the magnetodisk/current sheet morphology and its response to the various stresses on the disk plasma, and how these stresses vary in response to changes in the drivers of magnetospheric structure, both external (solar wind) and internal (Io mass-loading rate, global change in energetic particle population).

Further quantifying the relative importance of planetary rotation versus solar wind influence on the 
field and plasma configuration of the magnetodisk is an ongoing endeavor in the magnetospheric community. To support this aim, we require in situ observations of the system under a comprehensive range of solar wind conditions, as well as reliable tools for propagating solar wind parameters, observed in near-Earth space, out to the orbit of Jupiter (e.g., the mSWiM model, Zieger and Hansen [2008]). Further modeling and extensive field/ particle observations are also strongly required to fully characterize and understand the influence of plasma pressure anisotropy on the magnetodisk structure and stability. On the observational side, missions such as Juno (currently in orbit around Jupiter) and JUICE (JUpiter ICy moon Explorer, scheduled for launch in 2022) will be of great value in these pursuits.

\section{ACKNOWLEDGMENTS}

N. Achilleos was supported by the UK STFC Consolidated Grant (UCL/MSSL Solar and Planetary Physics, ST/N000722/1), and also acknowledges the support of the International Space Science Institute (ISSI), following useful discussions at ISSI Team Meetings "Coordinated Numerical Modeling of the Global Jovian and Saturnian Systems" and "Modes of Radial Plasma Transport in Planetary Systems." N. Achilleos is very grateful to both referees for their comments and suggestions.

\section{REFERENCES}

Achilleos, N., P. Guio, and C. S. Arridge (2010a), A MNRAS, model of force balance in Saturn's magnetodisc, Monthly Not. R. Astron. Soc., 401, 2349-2371.

Achilleos, N., P. Guio, C. S. Arridge, N. Sergis, R. J. Wilson, M. F. Thomsen, and A. J. Coates (2010b), Influence of hot plasma pressure on the global structure of Saturn's magnetodisk, Geophys. Res. Lett., 37, L20,201.

Achilleos, N., N. André, X. Blanco-Cano, P. C. Brandt, P. A. Delamere, and R. Winglee (2015), 1. Transport of mass, momentum and energy in planetary magnetodisc regions, Space Sci. Rev., 187, 229-299.

Bagenal, F. (2007), The magnetosphere of Jupiter: Coupling the equator to the poles, J. Atmos. Solar Terr. Phys., 69, 387-402.

Bagenal, F., and J. D. Sullivan (1981), Direct plasma measurements in the Io torus and inner magnetosphere of Jupiter, J. Geophys. Res., 86, 8447-8466.

Bagenal, F., and P. A. Delamere (2011), Flow of mass and energy in the magnetospheres of Jupiter and Saturn, J. Geophys. Res., 116, 5209.

Bagenal, F., R. L. McNutt, J. W. Belcher, H. S. Bridge, and J. D. Sullivan (1985), Revised ion temperatures for Voyager plasma measurements in the Io plasma torus, J. Geophys. Res., 90, 1755-1757.

Behannon, K. W., L. F. Burlaga, and N. F. Ness (1981), The Jovian magnetotail and its current sheet, J. Geophys. Res., 86, 8385-8401.
Bonfond, B., D. Grodent, J.-C. Gérard, T. Stallard, J. T. Clarke, M. Yoneda, A. Radioti, and J. Gustin (2012), Auroral evidence of Io's control over the magnetosphere of Jupiter, Geophys. Res. Lett., 39, L01, 105.

Caudal, G. (1986), A self-consistent model of Jupiter's magnetodisc including the effects of centrifugal force and pressure, J. Geophys. Res., 91, 4201-4221.

Connerney, J. E. P., M. H. Acuña, and N. F. Ness(1981), Modeling the Jovian current sheet and inner magnetosphere, J. Geophys. Res., 86, 8370-8384.

Cowley, S. W. H., and E. J. Bunce (2001), Origin of the main auroral oval in Jupiter's coupled magnetosphere-ionosphere system, Planet. Space Sci., 49, 1067-1088.

Goertz, C. K., B. A. Randall, M. F. Thomsen, D. E. Jones, and E. J. Smith (1976), Evidence for open field lines in Jupiter's magnetosphere, J. Geophys. Res., 81, 3393-3397.

Grodent, D., J.-C. Gérard, A. Radioti, B. Bonfond, and A. Saglam (2008), Jupiter's changing auroral location, J. Geophys. Res. Space Physics, 113, A01, 206.

Gurnett, D. A., and A. Bhattacharjee (2005), Introduction to Plasma Physics, Cambridge University Press, chap. 3.

Hill, T. W. (1979), Inertial limit on corotation, J. Geophys. Res., 25, 6554-6558.

Hill, T. W., A. J. Dessler, and C. K. Goertz (1983), Magnetospheric models, in Physics of the Jovian Magnetosphere, Cambridge University Press, 353-394.

Joy, S. P., M. G. Kivelson, R. J. Walker, K. K. Khurana, C. T. Russell, and T. Ogino (2002), Probabilistic models of the Jovian magnetopause and bow shock locations, J. Geophys. Res. Space Physics, 107, 1309.

Khurana, K. K. (1992), A generalized hinged-magnetodisc model of Jupiter's nightside current sheet, J. Geophys. Res., 97, 6269-6276.

Khurana, K. K. (1997), Euler potential models of Jupiter's magnetospheric field, J. Geophys. Res., 102, 11,295-11,306.

Khurana, K. K. (2001), Influence of solar wind on Jupiter's magnetosphere deduced from currents in the equatorial plane, J. Geophys. Res., 106, 25,999-26,016.

Khurana, K. K., and M. G. Kivelson (1993), Inference of the angular velocity of plasma in the Jovian magnetosphere from the sweepback of magnetic field, J. Geophys. Res., 98, 67-79.

Khurana, K. K., M. G. Kivelson, V. M. Vasyliunas, N. Krupp, J. Woch, A. Lagg, B. H. Mauk, and W. S. Kurth (2004), The configuration of Jupiter's magnetosphere, in Jupiter: The Planet, Satellites and Magnetosphere, edited by F. Bagenal, T. E. Dowling, and W. B. McKinnon, Cambridge University Press, 593-616.

Kivelson, M. G., and D. J. Southwood (2005), Dynamical consequences of two modes of centrifugal instability in Jupiter's outer magnetosphere, J. Geophys. Res., 110, A12,209.

Kivelson, M. G., et al (1997), Galileo at Jupiter: Changing states of the magnetosphere and first looks at Io and Ganymede, Adv. Space Res., 20, 193-204.

Nichols, J., and S. Cowley (2004), Magnetosphere-ionosphere coupling currents in Jupiter's middle magnetosphere: effect of precipitation-induced enhancement of the ionospheric Pedersen conductivity, Ann. Geophys., 22, 1799-1827.

Nichols, J. D. (2011), Magnetosphere-ionosphere coupling in Jupiter's middle magnetosphere: Computations including a 
self-consistent current sheet magnetic field model, J. Geophys. Res. Space Physics, 116, A10, 232.

Nichols, J. D., N. Achilleos, and S. W. H. Cowley (2015), A model of force balance in Jupiter's magnetodisc including hot plasma pressure anisotropy, J. Geophys. Res. Space Physics, 120, 10.

Paranicas, C. P., B. H. Mauk, and S. M. Krimigis (1991), Pressure anisotropy and radial stress balance in the Jovian neutral sheet, J. Geophys. Res., 96, 21.

Ray, L. C., and R. E. Ergun (2012), Auroral Signatures of Ionosphere-Magnetosphere Coupling at Jupiter and Saturn, American Geophysical Union, Geophysical Monograph Series 197, Washington, DC.

Ray, L. C., R. E. Ergun, P. A. Delamere, and F. Bagenal (2012), Magnetosphere-ionosphere coupling at Jupiter: A parameter space study, J. Geophys. Res. Space Physics, 117, A01, 205.

Scudder, J. D., E. C. Sittler, and H. S. Bridge (1981), A survey of the plasma electron environment of Jupiter: A view from Voyager, J. Geophys. Res., 86, 8157-8179.

Siscoe, G. L., A. Eviatar, R. M. Thorne, J. D. Richardson, F. Bagenal, and J. D. Sullivan (1981), Ring current impoundment of the Io plasma torus, J. Geophys. Res., 86, 8480-8484.
Smith, E. J., L. Davis Jr., D. E. Jones, P. J. Coleman Jr., D. S. Colburn, P. Dyal, C. P. Sonett, and A. M. A. Frandsen (1974), The planetary magnetic field and magnetosphere of Jupiter: Pioneer 10, J. Geophys. Res., 79, 3501-3513.

Southwood, D. J., M. K. Dougherty, P. Canu, A. Balogh, and P. J. Kellogg (1993), Correlations between magnetic field and electron density observations during the inbound Ulysses Jupiter flyby, Planet. Space Sci., 41, 919-930.

Thomas, N., F. Bagenal, T. W. Hill, and J. K. Wilson (2004), The Io neutral clouds and plasma torus, in Jupiter, The Planet, Satellites and Magnetosphere, Cambridge University Press, 561-591.

Thorne, R. M., T. P. Armstrong, S. Stone, D. J. Williams, R. W. McEntire, S. J. Bolton, D. A. Gurnett, and M. G. Kivelson (1997), Galileo evidence for rapid interchange transport in the Io torus, Geophys. Res. Lett., 24, 2131.

Vasyliūnas, V. M. (1983), Plasma distribution and flow, in Physics of the Jovian Magnetosphere, edited by A. J. Dessler, Cambridge University Press, New York, 395-453.

Zieger, B., and K. C. Hansen (2008), Statistical validation of a solar wind propagation model from 1 to $10 \mathrm{AU}, J$. Geophys. Res. Space Physics, 113, A08, 107. 